\title{
Les avantages de la méconnaissance de la francophonie : le cas de la Suède
}

\author{
Christophe Premat \\ Attaché de coopération linguistique et éducative auprès de l'Institut français de Suède \\ \& Chercheur associé au centre Émile Durkheim (Sciences Po Bordeaux, CNRS)
}

Les pays scandinaves ne font pas partie des aires francophones et sont marqués par une très forte maitrise de l'anglais et un potentiel multilingue que ne possèdent pas d'autres pays européens. Le français a eu une influence dans le passé au point d'être une langue obligatoire enseignée à l'école, mais cette langue fait partie des langues tierces enseignées dans ce pays au même titre que l'allemand et l'espagnol. Le dernier rapport sur la langue française en Suède officiellement remis au secrétaire général de l'Organisation Internationale de la Francophonie (O.I.F.) Abdou Diouf lors du dernier sommet de la francophonie à Montreux (22-24 octobre 2010) montre qu'il y a un recul d'environ 7\% des populations francophones en Europe alors que l'Afrique montre un essor considérable avec une augmentation de 7\% de locuteurs francophones (Diouf; Lane 94). Le rapport s'appuie uniquement sur les personnes pratiquant la langue au quotidien. Si la Suède n'est pas un pays francophone, il n'en existe pas moins une tradition francophile qui aménage des possibilités de valorisation de la francophonie. L'attribution du prix Nobel à Jean-Marie Gustave Le Clézio, écrivain franco-mauritien, a permis à la littérature francophone d'avoir ses lettres de noblesse.

Pour ce qui est des études francophones, on ne trouve pas de département à proprement parler, mais plusieurs départements de français ont inclus la littérature francophone dans l'offre de cours proposée. La problématique des études francophones émerge dans un contexte de remise en question des contenus culturels des cours. Cela se traduit en effet par une augmentation du nombre d'étudiants qui commencent à étudier le français dès leur entrée à l’Université.

Notre hypothèse est que les études francophones peuvent s'enraciner dans un contexte culturel qui méconnait relativement les possibilités du français en lien avec le monde francophone. Nous entendons par francophonie les pays où le français est une langue principale d'enseignement. Nous évoquerons la relation entre la situation de la langue française en Suède et l'intérêt pour le monde francophone ${ }^{1}$. Nous étudierons l'influence historique de la langue française en Suède puis nous dresserons un panorama des départements de langues suédois où il existe un intérêt pour les problématiques francophones ainsi que les travaux d'étudiants portant sur ce champ avant d'envisager les pistes de valorisation de la francophonie à l’Université en Suède.

\section{1) L'influence historique de la langue française en Suède}

La Suède a eu des influences du français au moment où elle se constituait comme nation importante. Le $17^{\mathrm{e}}$ siècle a vu des emprunts significatifs à la langue et aux codes culturels français. Les échanges intellectuels ont été intenses avec notamment la relation

\footnotetext{
${ }^{1}$ Nous préférons adopter cette démarche dans la mesure où tous les pays faisant partie de l'O.I.F. n'ont pas le même rapport à la langue française.
} 
entre Descartes et la reine Christine de Suède (Hochedez 2009). C'est l'époque où la défense de la langue suédoise se structure avec notamment l'institution de l'Académie Royale des Sciences de Suède en 1739 (Vetenskapakademien) et puis l'Académie Suédoise en 1786 (Svenska Akademien). Les emprunts à la langue française se multiplient d'autant plus que celle-ci était couramment utilisée par les familles royales d'Europe. À cette époque, des mots tels que möbel (meuble), balkong (balcon), garderob (garde-robe), salong (salon) $)^{2}$, parfym (parfum), mustasch (moustache), kastrull (casserole) font leur apparition dans la langue suédoise. La Révolution française eut un retentissement important en Suède, notamment dans certains cercles intellectuels (à l'instar du groupe Svensk Literatur Tidskrift animé par le philosophe Benjamin Höjer). De nombreux écrits politiques sont traduits au début du $19^{\mathrm{e}}$ siècle, faisant écho aux idées de la Révolution française (Perényi, 237)

Avec l'avènement de la dynastie des Bernadotte au début du $19^{\mathrm{e}}$ siècle, la culture française est à l'honneur. Cependant, l'idée selon laquelle Bernadotte aurait été à l'origine de transferts culturels importants entre la France et la Suède est erronée, puisque l'influence française était déjà assez conséquente au $18^{\mathrm{e}}$ siècle. $\mathrm{C}^{\prime}$ est pour sa politesse que Bernadotte a été remarqué : maréchal de l'Empire en 1804, il a fait capituler les Prussiens et a traité avec respect les officiers de la division suédoise fait prisonniers à Lübeck. C'est en partie grâce à ce comportement que la diète d'Örebro l'a élu comme prince héréditaire de Suède le 21 août 1810³. En 1813, il a rejoint la coalition contre la France avec des victoires décisives sur Oudinot à Gross-Beeren (23 août 1813) et Ney à Dennewitz (6 septembre 1813) (Bruyère-Ostells 43). Le 5 février 1818, il a succédé à Charles XIII, sous le nom de Charles XIV, roi de Suède et de Norvège. Il est l'ancêtre de nombreux monarques européens et a été à l'origine d'un fort sentiment francophile en Suède. L'enseignement du français a particulièrement progressé durant cette période : il est pratiqué dans les lycées en 1807, soit un siècle après son introduction dans les universités. En 1856, c'est même la première langue étrangère avant que l'allemand ne prenne le dessus en 1859 jusqu'à la Seconde Guerre mondiale ${ }^{4}$. Il y a eu plus d'emprunts à la langue française en suédois entre 1820 et 1859 que d'emprunts aux langues allemande et anglaise. Arthur Engberg, ministre de l'Instruction publique de Suède dans les années 1930, avait effectué une synthèse sur la situation des langues dans l'enseignement public en 1937 : le français y était facultatif, on pouvait l'apprendre en deuxième année de collège ; en revanche, "pour tous ceux qui se disposent à poursuivre leurs études dans les lycées et collèges, le français est obligatoire dans les deux dernières classes de l'école secondaire intermédiaire » (Östman, 46) ${ }^{5}$. Une telle position du français a évidemment changé, puisque le français $n^{\prime}$ est plus depuis longtemps une langue obligatoire dans l'enseignement public suédois. L'anglais est la seule langue obligatoire au niveau du collège et le français est

\footnotetext{
2 Selon le dictionnaire de l'académie suédoise, le terme est apparu en 1778 dans la langue suédoise. Le mot parfym est ajouté en 1797 par exemple.

${ }^{3}$ Les communes d’Örebro et de Pau ont célébré le bicentenaire de cet événement le 21 août 2010. Pau abrite un musée Bernadotte et ces deux communes sont jumelées.

${ }^{4}$ Ibid., p. 45. En 1905, le français reculait à la troisième place après l'allemand et l'anglais. Après la Seconde Guerre mondiale, l'anglais a pris progressivement la place de l'allemand. Le français est une langue choisie majoritairement par les filles puisque 80\% des écoles de jeunes filles en 1888 dispensent un enseignement de français.

${ }^{5}$ Margareta \& Hans Ö́stman, Au Champ d'Apollon, Écrits d'expression française produits en Suède (1550-2006), Filologiskt arkiv 47, Stockholm 2008, p. 46.
} 
aujourd'hui une langue facultative, souvent choisie à titre de troisième langue en Suède ${ }^{6}$.

\section{2) Les études francophones en Suède}

Avant de passer en revue le périmètre dévolu aux études francophones en Suède, il importe de donner une idée des publics étudiant la langue française en Suède. Le rapport de l'OIF de l'automne dernier fait ressortir une comparaison entre les pays scandinaves et nordiques. Les conquêtes de nouveaux publics en français sont un défi pour les départements de français et plus généralement pour les instituts et associations culturelles liés à la francophonie en Suède.

a) Éléments de comparaison

\begin{tabular}{|l|l|l|l|}
\hline $\begin{array}{l}\text { Nombre d'élèves } \\
\text { suivant un parcours } \\
\text { francophone }\end{array}$ & Primaire & Secondaire & Supérieur \\
\hline $\begin{array}{l}\text { Norvège (2007- } \\
\text { 2008) } \\
\text { Population : 5 M }\end{array}$ & $150-200$ & 47633 & 250 \\
\hline $\begin{array}{l}\text { Danemark (2008- } \\
\text { 09) } \\
\text { Population : 5,5 M }\end{array}$ & Env. 200 & 20600 & 313 \\
\hline $\begin{array}{l}\text { Suède (2007-2008) } \\
\text { Population : 9 M }\end{array}$ & 2000 & 136000 & 2500 \\
\hline $\begin{array}{l}\text { Islande (2007-2008) } \\
\text { Population : } \\
312.000\end{array}$ & 2554 & 63 \\
\hline $\begin{array}{l}\text { Finlande (2008- } \\
\text { 2009) } \\
\text { Population : 5,2 M }\end{array}$ & 6153 & 26278 & 11200 \\
\hline
\end{tabular}

Sources : La langue française dans le monde 2010, Paris, éditions Nathan, 2010, p. 154

Nous avons ici une estimation du nombre d'élèves poursuivant un parcours francophone dans le primaire, le secondaire et le supérieur dans les pays nordiques et scandinaves. La Finlande a des résultats impressionnants du fait du réseau de classes bilingues permettant un suivi des élèves dès le primaire d'où le nombre élevé de publics étudiant dans un parcours francophone à l'université. La Suède dispose de publics d'apprenants importants dans le secondaire, le français restant une langue de référence parmi les langues tierces. Depuis 2009, des classes bilingues ont émergé avec environ 500 élèves concernés qui étudient le français non plus uniquement comme langue étrangère mais comme langue de communication.

Les départements d'études francophones sont confrontés à une augmentation sensible du nombre d'étudiants de niveau débutant, d'où l'intérêt pour eux de réfléchir à un lien plus fort avec les lycées (Premat 2009). Si le nombre de débutants augmente, il

${ }^{6}$ Christophe Premat, «Les relations culturelles franco-suédoises », Nordiques, No22, Printemps-été 2010, pp. 79-92. 
devient difficile de fidéliser des publics susceptibles de poursuivre des études longues de langue française. La crise que traversent les départements de français est un défi qui contraint les acteurs du français au niveau universitaire à imaginer de nouveaux contenus culturels. Le Ministère des Affaires étrangères et européennes français avait initié une réflexion sur le devenir des départements de français à l'étranger en 2009 pour évoquer les manières d'attirer de nouveaux publics ${ }^{7}$. Les responsables des départements de français des universités à l'étranger avaient alors été invités pour échanger leurs points de vue sur l'avenir des études de français. C'est le troisième séminaire qui porte sur ce sujet depuis le début des années 2000. La valorisation des études francophones pourrait selon nous être de nature à renouveler l'approche des départements de français trop concentrés sur la linguistique. "En Suède, le français acquiert ses lettres de noblesse à l'Université d'Uppsala en 1637 grâce au maître des langues (Språkmästare), Isaac Cujacius. Un ouvrage de didactique du français est publié par Alexandre du Cloux en 1646. Aux $19^{\mathrm{e}}$ et $20^{\mathrm{e}}$ siècles, les études universitaires de français se structurent à Uppsala autour de Per Adolf Geiger (1841-1919), d'Anna Ahlström (1863-1949), cette dernière ayant été la première femme à obtenir le grade de docteur en linguistique. À Lund, la didactique du français a été influencée au $19^{\mathrm{e}}$ siècle par des grands spécialistes tels qu'Emanuel Olde (1802-1885) et l'université de Göteborg possède d'éminents linguistes (Karl Michaëlsson). L'École de Stockholm s'est structurée après la Seconde Guerre mondiale avec notamment Bertil Maler, Ake Blomqvist et Gustaf Holmér. Si l'on résume l'évolution de la didactique du français en Suède, on peut constater que le $19^{\mathrm{e}}$ siècle a été marqué par des professeurs de langue aux compétences généralistes tandis que le $20^{\mathrm{e}}$ siècle a vu émerger des profils spécialisés de professeurs et de chercheurs en linguistique »(Premat 2009). Les premiers linguistes étaient bien plus généralistes avant que les études de français ne se spécialisent davantage.

b) Les études francophones en Suède

Il n'existe pas de département institutionnel ayant un tel intitulé en Suède, mais les études francophones restent disséminées dans les départements de langues romanes. Des thèses récentes ont été soutenues en Suède montrant un intérêt grandissant pour la francophonie à l'Université (Voir Carlshamre, Kilito, Lindberg). Plusieurs appels à candidature à des postes de professeur d'Université s'ouvrent avec une mention relative à la littérature francophone. L'Institut des études canadiennes est lié à l'Université de Stockholm et est dirigé par l'actuelle présidente de l'association des professeurs de français de Suède.

Des rencontres avec des écrivains francophones sont organisées dans le cadre de séminaires obligatoires pour les étudiants. L'année 2009, Rita Mestokosho, écrivaine de langue innue nommée dans le discours de réception du prix Nobel de Le Clézio, est venue participer à des ateliers pédagogiques et à des conférences à l'Université. ${ }^{\prime}$ La couverture médiatique a été large puisque plusieurs articles de journaux nationaux

\footnotetext{
${ }^{7}$ Les actes du colloque sont disponibles sur le site du CIEP. http://www.ciep.fr/conferences/evolutiondes-departements-etudes-francaises-des-universites-europeennes/docs/situation-et-perspectives-devolution-des-departements-d-etudes-francaises-des-universites-europeennes/index.html [Site consulté le 18 août 2011]. 8 http://www.latitudefrance.org/Rencontre-avec-Rita-Mestokosho.html [Site consulté pour la dernière fois le 17 août 2011].
} 
Dagens Nyheter et Svenska Dagbladet lui ont été consacrés. Les éditions Beijboom books de Göteborg ont alors osé éditer ses ouvrages en édition multilingue en français, anglais et innu puis en suédois, innu et français. Rita Mestokosho est intervenue lors de journées pédagogiques à l'école franco-suédoise et a fait partie du programme de conférences du département de français de l'Université de Stockholm. Edem Awumey, écrivain francophone d'origine togolaise et résidant au Québec, est venu également donner des conférences sur son œuvre et a participé au salon du livre 2010 à Göteborg dont le thème était l'Afrique. Une table-ronde avait été organisée sur la francophonie et son œuvre et il a accordé un entretien qui a été publié sur la revue Sens Public 9 . Toutes ces manifestations ont eu une résonance au sein du public suédois et la francophonie a pu être mise à l'honneur.

Il semble que la francophonie émerge comme nouvel objet d'études au sein du champ intellectuel et universitaire suédois, mais la greffe ne pourra prendre qu'à trois conditions selon nous. Premièrement, il faut d'abord une inclusion des manifestations francophones extérieures organisées avec le concours d'instituts et d'associations culturels dans le programme de cours et de recherches des étudiants. En second lieu, les départements de français doivent faciliter une approche résolument transdisciplinaire favorisant les traductions entre le français et suédois ; il est primordial que les publics aient accès aux contenus dans les deux langues pour ne pas exclure des publics limités par la maitrise de la langue française. Troisièmement, les départements doivent nouer des contacts plus étroits avec les institutions travaillant sur l'Afrique et le Canada qui constituent deux viviers importants pour la francophonie. Pour l'Afrique, l'Institut nordique des études africaines d'Uppsala constitue un partenaire incontournable tout comme le centre d'études africaines de l'Université de Göteborg et pour le Canada, l'Institut des études canadiennes et la représentation suédoise de l'association internationale des études québécoises.

Plusieurs colloques portant sur la littérature francophone ont eu lieu en Suède pour dynamiser les études francophones, mais la majorité des postes concerne des chercheurs et des professeurs de linguistique. Les stratégies interdisciplinaires pourraient permettre de valoriser les études francophones. Il existe des tentatives intéressantes mais qui restent relativement embryonnaires à l'instar du département de philosophie de l’Université de Södertörn qui travaille en partie sur la philosophie française $^{10}$. Le concept de littérature-monde est également proposé pour tenter d'y adjoindre une perspective sur la francophonie même si les notions de francophonie et de World-Literature ne sont pas superposables ${ }^{11}$.

\section{3) Les stratégies de conquête de nouveaux publics en Suède}

\footnotetext{
${ }^{9}$ Christophe Premat, «Les espaces anonymes, entre voyage et exil. Entretien avec l'écrivain Edem Awumey » http://www.sens-public.org/spip.php?article845, 6 juin 2011.

10 http://www.latitudefrance.org/Les-emigres-grecs-et-leur.html Un ouvrage est en préparation aux éditions du Manuscrit (Christophe Premat, Servanne Jollivet, Mats Rosengren, Destins d'exilés. Trois philosophes grecs á Paris : Kostas Axelos, Cornélius Castoriadis, Kostas Papaioannou). Les événements de ce type permettent à plusieurs départements universitaires de travailler sur des penseurs francophones.

${ }^{11}$ http://www.latitudefrance.org/Colloque-sur-la-litterature-monde.html Conférence organisée le 20 mai 2011 à l’Université de Stockholm. Publication du compte-rendu sur le site du réseau culturel français dans le monde. [Site consulté pour la dernière fois le 17 août 2011].
} 
La Suède est un pays non francophone qui a vu le taux d'apprenants du français diminuer de manière drastique au début des années 2000. Le nombre d'apprenants s'est stabilisé en 2008 en raison de nouvelles lois valorisant les coefficients des langues vivantes. Le contexte défavorable incite les acteurs du français à imaginer des manières originales d'attirer les publics vers le français ${ }^{12}$. La stratégie du Ministère des Affaires étrangères et européennes français est de promouvoir le multilinguisme pour renforcer le statut du français comme langue incontournable.

\section{a) Promouvoir des événements liés à la francophonie}

L'influence de la langue française est certaine en Suède, mais la francophonie en tant que promotion de la diversité culturelle autour de la langue française reste mal connue. Le terme Frankofoni existe en suédois, mais il constitue un néologisme calqué sur l'usage du mot en français. Les publics suédois ont été habitués à une image du rayonnement de la culture française des années 1960 et commencent à s'intéresser aux aires francophones en général. Il existe des instituts de recherche travaillant sur les pays francophones sans que le français soit utilisé comme langue de travail à l'instar de l'agence suédoise nationale pour le développement (SIDA) ${ }^{13}$. La francophonie est ignorée de ce pays qui n'a aucun contact particulier avec l'OIF. L'OIF a eu une politique d'élargissement notoire ces dernières années en passant de 56 États membres en 2008 à 75 États en 2011. Certes, une grande partie de ces nouveaux adhérents sont observateurs ou invités, mais l'OIF souhaite consolider son réseau d'États pour y faire valoir ses options politiques en matière de gouvernance démocratique.

La promotion du français consiste davantage à créer des événementiels importants pour susciter l'envie d'apprendre cette langue qui reste importante. Le concours de la francophonie a été lancé en 2009 en partenariat avec l'Institut français de Stockholm, les services culturels de l'ambassade de France en Suède, l'association des professeurs de français de Suède, la section française de l'association des professeurs de langues vivantes de Suède ainsi qu'un certain nombre d'ambassades francophones. Les dix mots choisis chaque année pour la francophonie sont l'occasion de faire travailler les apprenants suédois et d'organiser des manifestations autour de la journée internationale du 20 mars. Cette politique vise à la fois à motiver les apprenants, mais surtout à encourager les représentants des départements universitaires de français à renouveler leurs approches ${ }^{14}$. Un site à l'usage des professeurs et des apprenants a été lancé à la fin de $2009^{15}$ car la promotion par les nouvelles technologies est essentielle dans un pays où plus de $90 \%$ des habitants sont connectés à Internet. Chaque année, un concours de la francophonie est organisé en Suède avec une remise des prix autour du 20 mars en présence des ambassadeurs francophones. Le dernier concours portait sur des productions de rap illustrant les dix mots ou des essais : les meilleures prestations ont été primées et mises en ligne ${ }^{16}$. L'objectif de l'Institut et des associations de professeurs de langues vivantes est de créer des événements réguliers afin que les professeurs

\footnotetext{
12 Note sur la situation du français en Suède en 2009, ambassade de France en Suède.

13 http://www.sida.se [Site consulté le 22 août 2011].

${ }^{14}$ http://www.franskaspraket.eu/spip.php?article78 Site du concours de la francophonie 2011 en Suède. [Site consulté pour la dernière fois le 20 août 2011].

${ }^{15} \mathrm{http}: / /$ www.franskaspraket.eu [Site portant sur la langue française en Suède et lancé par l'Institut français de Stockholm en décembre 2009 en collaboration avec la revue Sens Public].

${ }^{16}$ http://www.franskaspraket.eu/spip.php?article85 [Site consulté pour la dernière fois le 20 août 2011].
} 
puissent se les approprier et les intégrer à leur plan d'études. À côté de la francophonie, le concours des Olympiades de langues (Språkolympiaden) est un moment important de la promotion des langues avec une méthode adaptée aux écoles suédoises puisque les élèves participent à des quizz portant sur la langue et la culture. Les Olympiades concernent les langues tierces, l'espagnol, l'allemand et le français et sont une collaboration entre l'Institut français, le Goethe et le Cervantès. Les épreuves et les quizz sont imaginés par les élèves avec plusieurs étapes (quarts de finale, demi-finales régionales et finale nationale) et deux catégories (collèges et lycées). Cette année, plus de 7000 élèves et 130 écoles ont participé à ces Olympiades qui sont fortement médiatisées avec des sponsors fidèles (Chambre de Commerce et d'Industrie du sud de la Scanie.... $)^{17}$. Les Olympiades ont lieu dans les écoles et les lycées et ne sont pas extérieures aux cours de langues; les quizz encouragent la curiosité des élèves qui se préparent à ces épreuves en classe. Il est prévu une extension de ce projet à l'ensemble de la Scandinavie. Le jeu reste une dimension essentielle de l'apprentissage des langues et ce type de compétition ludique montre aux élèves qu'ils peuvent se dépasser pour faire gagner leur groupe ou leur classe ${ }^{18}$.

En effet, plusieurs séminaires ont montré le changement des études francophones du fait de nouveaux publics d'apprenants. De plus en plus d'étudiants commencent à étudier le français à l'Université et très peu d'entre eux continuent à un niveau plus avancé. Cela a une influence sur le contenu même des cours et des intitulés puisque l'offre est davantage tournée vers du Français Langue Étrangère. Les cours de civilisation francophone sont également importants, mais une grande majorité des départements francophones des universités scandinaves reste dominée par les études linguistiques au détriment de la littérature et des études culturelles. Il existe actuellement une dizaine de départements de français en Suède répartis dans les villes suivantes: Växjö-Kristianstad, Stockholm, Uppsala, Lund, Göteborg, Linköping, Falun et Umeå. Les études avancées de linguistique prédominent dans ces départements dont certains ont fermé depuis le début des années 2000 à l'instar de ceux d’Örebro, de Skövde et de Karlstad. Certains départements doivent leur survie grâce à la présence d'autres langues, les langues romanes donnant une assise plus forte aux études de français.

Le renouvellement des contenus culturels serait certainement de nature à favoriser un autre type d'intérêt pour les pays francophones. De plus, le cloisonnement des départements de langue ne permet pas au français de pénétrer les autres champs professionnels alors même que le Français Langue Étrangère est marqué par cette recherche avec le développement du Français sur Objectifs Spécifiques. Les départements de langue gagneraient à travailler davantage en synergie avec d'autres matières pour renforcer leur attractivité. Il devient primordial d'attirer des publics s'intéressant concrètement au français comme langue de communication. Au sein des départements de langues romanes, les études francophones constitueraient sans aucun doute un pôle fort d'attractivité et de rayonnement.

b) Le français professionnel

\footnotetext{
17 http://www.latitudefrance.org/Finale-des-Olympiades-de-langues-a.html

${ }^{18}$ Les témoignages des professeurs ayant accompagné les élèves dans leurs productions ont été publiés en ligne. http://www.franskaspraket.eu/spip.php?article83 Les fiches pédagogiques des professeurs ont été publiées sur le site de l'association des professeurs de français de Suède. Voir http://www.fransklararforeningen.com/ [Site consulté pour la dernière fois le 20 août 2011].
} 
La langue reste un moyen pour valoriser d'autres compétences et pas forcément une finalité en $\operatorname{soi}^{19}$. Le Ministère des Affaires étrangères et européennes français a lancé un portail centré sur le français professionnel intitulé «Oui, je parle français dans mon entreprise » comportant des faits, des analyses et des événements sur l'usage du français dans un milieu professionnel ${ }^{20}$. Le français a des usages concrets et sort de l'apprentissage artificiel déconnecté des trajectoires professionnelles des individus. Un colloque a eu lieu le 12 mai 2011 à l'école de commerce de Stockholm sur le multilinguisme et la vie en entreprise ${ }^{21}$. Il a été organisé par l'Institut français, le Goethe Institut, l'Institut Cervantes, le Centre culturel italien, la chambre de commerce italosuédoise et l'entreprise. Plusieurs langues ont été utilisées, le colloque a permis d'avoir des discussions sur les avantages du multilinguisme et de la traduction dans le milieu de l'entreprise. La Suède présente un paradoxe: si la population est majoritairement multilingue, les petites et moyennes entreprises fondent leur stratégie internationale uniquement sur l'anglais et perdent ainsi des marchés à l'exportation ${ }^{22}$.

Il faut décomplexer l'usage du français qui a toujours cette image d'une langue d'accès difficile pour permettre à ces publics débutants de poursuivre leurs études et leurs recherches sur les aires francophones. Il est ainsi important d'attirer des économistes et des politologues par exemple vers l'étude des pays francophones en leur donnant un niveau de langue satisfaisant (Faramond, 227). En d'autres termes, plus la langue sera un outil pour la culture, plus les départements de français arriveront à diversifier leurs contenus ${ }^{23}$.

c) La relance des certifications, un gage de qualité

Les certifications de français peuvent être également un moteur d'apprentissage pour des étudiants souhaitant compléter leur parcours professionnel en France par la suite. Certes, le contexte éducatif suédois demeure assez hostile à l'implantation d'une culture du diplôme, mais l'avantage du Diplôme d’Études de Langue Française (DELF) est qu'il permet aux étudiants de situer leurs compétences langagières (Premat 2010, $133)^{24}$. Le cadre européen commun de référence pour les langues a été récemment importé en Suède et permet de dynamiser l'apprentissage des langues et leur évaluation. Depuis l'adaptation des examens de DELF et de Diplôme Approfondi de Langue

\footnotetext{
${ }^{19}$ Entretien avec Bienvenu Sene Mongaba à l'Institut français de Stockholm, 30 septembre 2010 (L'entretien paraitra à l'automne 2011 sur la revue Sens Public).

20 http://www.ouijeparlefrancais.com/

${ }^{21}$ http://www.latitudefrance.org/Pour-le-12-Mai-conference.html

22 http://www.franskaspraket.eu/spip.php?article96 Article en suédois d’Ingela Bel Habib sur la relation entre langues étrangères et exportation des entreprises [Site consulté pour la dernière fois le 20 août 2012]. Voir aussi le projet du ministère du commerce extérieur suédois sur la stimulation du réseau de chefs d'entreprise multiculturels. http://www.ouijeparlefrancais.com/le-multilinguisme-en-europe-etles, 602.html [article datant du 22 novembre 2010 et consulté pour la dernière fois le 20 août 2011. Ce projet suédois se prénomme « Kosmopolit»].

${ }^{23}$ Voir l'étude danoise à ce sujet sur les langues étrangères. Lisbeth Verstraete Hansen, Robert Philipson, Fremmedsprog til fremtiden, Sprogpolitiske udfordringer for Danmark, École de commerce de Copenhague, 2008, http://www.sprakforsvaret.se/sf/fileadmin/PDF/Fremmedsprog for fremtiden.pdf [Site consulté le 20 août 2011].

${ }^{24}$ Christophe Premat, Véronique Simon, «Les certifications ont-elles un effet décisif sur l'apprentissage du français? ", Synergies Pays Scandinaves, nº5, 2010, pp. 133-142. Voir http://www.latitudefrance.org/Ledeveloppement-des.html sur l'augmentation des certifications de français en Suède.
} 
Française (DALF) ${ }^{25}$ au cadre européen commun de référence, il y a une progression modeste de ces certifications en Suède. L'évaluation des élèves et la formation des professeurs sont un débat important, le système éducatif suédois étant en pleine transformation en $2011^{26}$. Les élèves suédois ne reçoivent des notes qu'à partir des deux dernières années de collège, ils ne redoublent pas, l'éducation se fondant sur l'égalité des chances, le respect du groupe et des valeurs démocratiques. Il existe un relatif consensus pour introduire plus tôt la culture de l'évaluation afin que les compétences des élèves soient davantage valorisées. L’Institut français de Stockholm profite de cette opportunité pour implanter le DELF et montrer son utilité dans le cadre d'une mobilité professionnelle ou étudiante vers un pays francophone.

Il existe une réflexion importante dans les départements de français des pays scandinaves et nordiques pour comprendre les raisons du déclin des étudiants se spécialisant en langue française. Si le diagnostic commence à être accepté, les solutions restent encore discutées. Cette situation devient une opportunité à saisir comme en Suède où la francophonie reste un domaine encore mal connu. Cette curiosité pourrait être davantage satisfaite par une solidarité des acteurs du français, qu'ils soient associatifs, universitaires et scolaires afin de créer de véritables départements d'études francophones. Dans un pays connu pour son aide conséquente pour les pays en voie de développement, la promotion de la francophonie pourrait s'avérer un investissement culturel et intellectuel fiable sur le long terme. La grande époque du français (stora tider en suédois) doit laisser place à celle de la francophonie et du multilinguisme. Il est pour cela nécessaire d'attirer les élèves vers une aire culturelle plus vaste, celle des pays francophones pour encourager l'apprentissage de la langue (Lane 99). Paradoxalement donc, la méconnaissance actuelle de ces pays est plutôt un atout pour renforcer l'attractivité de la langue française en Suède.

\footnotetext{
${ }^{25}$ http://www.ciep.fr/delfdalf/ [Site consulté le 21 août 2011]

${ }^{26}$ Voir l'onglet «Système éducatif suédois » sur le site www.franskaspraket.eu qui explique les différentes réformes du système éducatif suédois. La revalorisation du métier d'enseignant devrait avoir un impact sur l'apprentissage des langues.
} 


\section{Bibliographie}

Bruyère-Ostells, Walter. "Les officiers républicains sous l'Empire: entre tradition républicaine, ralliement et tournant libéral », Annales historiques de la Révolution française 346 (2006) : 43.

Carlshamre, Katarina. Pulsion et résistance : Émancipation, liberté et tendances conservatrices dans trois romans d'Anne Hébert, Département de français/italien de l'Université de Stockholm, 2009.

Diouf, Abdou. La langue française dans le monde. Paris : éditions Nathan, 2010.

Faramond, Guy (de). Svea \& Marianne, Les relations franco-suédoises, une fascination réciproque. Paris : éditions Michel de Maule, 2007.

Hochedez, Camille. " "Bienvenue au pays du Nja" : la Suède est-elle eurosceptique ?» Café Géo, 28 juin 2009, http:/ / www.cafe-geo.net/article.php3?id article=1621

Kilito, Sarah. Femmes-sujets : Étude sur les auvres de romancières marocaines francophones entre 1982 et 1999, Département de français et d'italien de l'Université de Stockholm, 2004.

Lane, Philippe. Présence française dans le monde, l'action culturelle et scientifique. Paris : La Documentation Française, 2011.

Lindberg, Svante. Pratiques de l'ici, altérité et identité dans six romans québécois des années 1989. 2002, Département de français et d'italien de 1’Université de Stockholm, 2005.

Mestokosho, Rita. Hur jag ser på livet mormor, Eshi Uapataman Nukum/How I see life, grandmother, Eshi Uapataman Nukum/Comment je percois la vie de grand-mère. Göteborg : Beijboom Books AB, 2011.

Östman, Margareta \& Hans. Au Champ d'Apollon, Écrits d'expression française produits en Suède (1550-2006). Filologiskt arkiv 47, Stockholm 2008.

Perényi, Janos. «La Révolution française et la Suède », Annales historiques de la Révolution française 277 (1989) : 237-243.

Premat, Christophe, \& Véronique Simon, «Les certifications ont-elles un effet décisif sur l'apprentissage du français? ? Synergies Pays Scandinaves 5 (2010) : 133-142.

Premat, Christophe. «Rapport sur l’histoire comparée des études de français dans les pays scandinaves et nordiques », Bulletin électronique de l'ADIT, 18 novembre 2009, http://www.bulletins-electroniques.com/actualites/61272.htm.

Sites web consultés :

http://www.latitudefrance.org/Jean-Marie-Le-Clezio-Prix-Nobel-de.html [consulté le 17 août 2011].

http://www.latitudefrance.org/Rencontre-avec-Rita-Mestokosho.html [consulté le 17 août 2011].

http://www.latitudefrance.org/Trois-questions-posees-a-Edem.html [consulté le 17 août 2011].

http://www.emilangues.education.fr/international/emile-clil-europe/finlande Site sur l'éducation plurilingue à partir de l'enseignement de matières par une langue étrangère intégrée. [Site consulté le 20 août 2011]. 